\title{
In memoria di Sandro Geatti
}

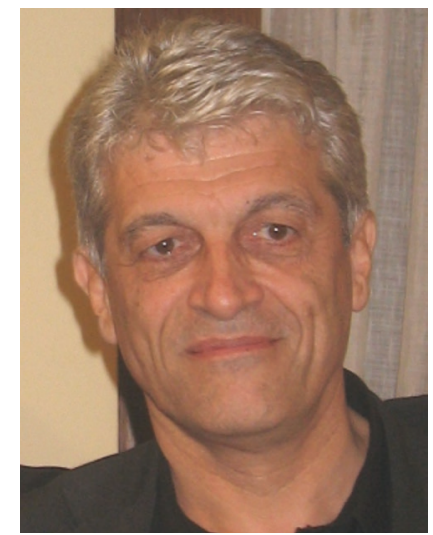

Sandro Geatti
Con Sandro se ne va una parte importante del mio percorso professionale.

Lo ricorderò sempre per la sua correttezza, per la sua eleganza professionale, per le sue idee innovative, soprattutto quelle dedicate alla 'sua' Filiale Italiana dell'EDTNA/ERCA.

Quando un altro importante e notevole infermiere dello scenario italiano della nefrologia e dialisi, Tiziano Cerrai, mi fece conoscere Sandro all'inizio degli anni '90, ebbi subito l'impressione di un uomo giusto, riflessivo, colto, capace di prendere positivamente per mano e crescere assieme a quel nugolo di infermiere (erano quasi tutte femmine ... antesignane di quel che sarebbe successo e succederà ancor di più in un prossimo futuro) che mi sembrarono professionalmente così avanzate e progressiste da farmi pensare per un certo periodo di aver sbagliato strada a fare il medico: avrei voluto essere infermiere in quel gruppo!

Con Sandro ricordo alcuni viaggi a Roma all'allora Ministero della Sanità, per qualche progetto probabilmente troppo avanzato per essere accettato, quando in viaggio mi raccontava della sua esperienza nel Regno Unito.

Insieme a Sandro e con la allora Filiale Italiana EDTNA/ ERCA, oggi confluita in SIAN, siamo stati protagonisti di tanti congressi - tra cui certamente Barcellona- ed abbiamo contribuito con entusiasmo ed orgoglio a tanti lavori e pubblicazioni. Ricordo in particolare il libretto sulle Raccomandazioni $\mathrm{HCV}$ in Dialisi.

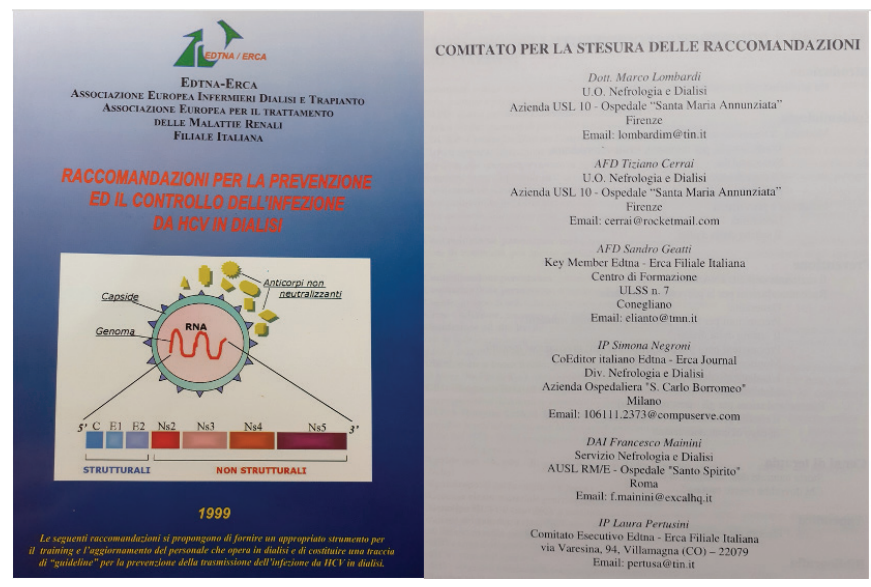

Sandro e i 'suoi' hanno collaborato molto con il GTN\&D, oggi GCND. SIAN è tutt'ora uno dei tre principali soggetti associativi con cui collabora con il giornale. I più recenti contributi di rilievo di Sandro al nostro giornale risalgono al 2017 e al 2018, con le prime due parti di quella che doveva essere una trilogia dedicata ad Un secolo di Nursing Nefrologico. La malattia lo ha costretto ad abbandonare questo progetto, probabilmente per non rubare tempo agli affetti a lui più cari.

Sandro mi mancherà molto ma penso che mancherà soprattutto alle più giovani leve infermieristiche che non potranno aver più in lui un'icona di come, nella seconda parte del secolo scorso, sono stati i grandi infermieri come lui, quelli che contavano e su cui si poteva sempre contare.

\section{Marco Lombardi \\ Editor in Chief}

Received: January 3, 2021

Accepted: January 14, 2021

Published online: February 18, 2021

Indirizzo per la corrispondenza:

Marco Lombardi

Ospedale del Mugello

Via Della Resistenza, 60

50032 Borgo San Lorenzo, Firenze - Italia

lombardim@tin.it 